\title{
ON COHOMOLOGICAL DEFORMATIONS OF BICROSSED PRODUCT HOPF ALGEBRAS
}

\author{
JeONg HeE HoNg ANd WoJCIECh SZYMAŃSKI
}

\begin{abstract}
We construct and describe two classes of examples of twisted bicrossed product Hopf algebras corresponding to matched pairs of finite groups.
\end{abstract}

\section{INTRODUCTION}

In this article we describe in detail two methods of producing finite dimensional Hopf algebras by deforming bicrossed products corresponding to matched pairs of finite groups. Our motivation to study these constructions has two sources. Firstly, the classification of finite dimensional Hopf algebras is not yet in sight and their theory is still in the stage of finding new ways of building the objects in question. Secondly, there is an interesting interplay between the theory of Hopf *-algebras (or Kac algebras) and the Jones index theory of subfactors. Namely, subfactors of depth 2 correspond to crossed products of Kac algebras. Furthermore, it has been shown recently [3, 4] that a depth 2 inclusion of the type $\mathbf{P}^{\mathbf{K}} \subseteq \mathbf{P} \times \mathbf{H}$, with $\mathbf{K}, \mathbf{H}$ finite groups acting outerly on a factor von Neumann algebra $\mathbf{P}$, corresponds to a cohomologically deformed bicrossed product Kac algebra related to a matched pair of groups $\mathbf{G}=\mathbf{K H}$ [6].

We briefly outline such a deformation. If $\mathbf{G}=\mathbf{K H}$ is a matched pair of groups and $k$ is a field then there is a natural way to define a Hopf algebra structure in the crossed product algebra $k(\mathbf{K}) \rtimes \mathbf{H}[\mathbf{6}]$. The multiplication and comultiplication of the resulting bicrossed product Hopf algebra $\mathbf{K} \bowtie \mathbf{H}$ may be deformed by means of suitably matched 2-cocycles $\mu: \mathbf{H} \times \mathbf{H} \times \mathbf{K} \rightarrow k^{*}$ and $\nu: \mathbf{K} \times \mathbf{K} \times \mathbf{H} \rightarrow k^{*}$. This construction goes back to $\mathrm{Kac}[5]$ and is essentially equivalent to the problem of finding all $k$-Hopf algebra extensions of $k(\mathbf{K})$ by $\mathbf{H}$. Even though this problem was solved in principle by Kac in terms of cohomology of his double complex associated to the matched pair, concrete non-trivial examples are difficult to handle due to excessively hard calculations $[\mathbf{7}, \mathbf{8}]$. The crux of the problem lies in solving the key equation (2) of the present article. We

Received 28th January, 1999

The first author wishes to acknowledge the financial support of the Korea Research Foundation made in the program year of 1997 . The second author was partially supported by an RMC University of Newcastle visitor grant 45/290/754, Australian Research Council, Australian Academy of Science, and Korea Science and Engineering Foundation.

Copyright Clearance Centre, Inc. Serial-fee code: 0004-9727/99 \$A2.00+0.00. 
believe that Proposition 1 singles out the easiest obtainable non-trivial solutions. This we demonstrate in Examples 3 and 4, which deal with matched pairs $\mathbf{S}_{4}=\mathbf{D}_{\mathbf{4}} \mathbf{Z}_{3}$ and $\mathbf{P S L}\left(2, \mathbf{F}_{7}\right)=\left(\mathbf{Z}_{7} \rtimes \mathbf{Z}_{3}\right) \mathbf{D}_{4}$, respectively. The latter example involves a matched pair decomposition of a simple group.

Recently much progress has been made in finding deformations of finite dimensional Hopf algebras by means of pseudococycles $[\mathbf{1}, \mathbf{9}, \mathbf{1 0}, \mathbf{1 1}]$. In brief this method works as follows. If $\mathbf{A}$ is a Hopf algebra then one tries to find an invertible $\Omega \in \mathbf{A} \otimes \mathbf{A}$ such that $\mathbf{A}$ with the old multiplication and a new comultiplication $\Delta_{\Omega}(x)=\Omega \Delta(x) \Omega^{-1}$ is a Hopf algebra. Technical difficulties in constructing non-trivial pseudococycles $\Omega$ are formidable. In Theorem 7 of the present article we construct a class of pseudococycles for bicrossed product Hopf algebras built on semi-direct product groups $\mathbf{G}=\mathbf{K} \rtimes \mathbf{H}$ with $\mathbf{H}$ Abelian.

One of the most natural invariants used in distinguishing non-isomorphic Hopf algebras is the intrinsic group of group-like elements. An $x \neq 0$ is group-like if $\Delta(x)=x \otimes x$. Group-like elements are also important in the investigations of towers of subfactors [2]. For both constructions discussed in this paper we describe group-like elements of the resulting Hopf algebras (see Proposition 2 and Theorem 9). The result of Proposition 2 is not actually new. It was originally proved in [3, Proposition 2.2]. We feel that the present argument is much cleaner, more illuminating and, in addition, similar to the one used in the proof of Theorem 9.

In closing let us mention three open problems which naturally arise from the present article. Firstly, the precise relationship between the two deformations from Proposition 1 and Theorem 7 is not clear to us at the moment. Secondly, we feel that removal of some simplifying assumptions of Theorem 7 ( $\mathbf{H}$ Abelian, $\mathbf{G}$ a semi-direct product) should be possible. Thirdly, it would be interesting to find a subfactor realisation of the construction from Theorem 7 . This would perhaps be analogous to the composition of subfactors relevant to the 2-cocycle deformations of bicrossed products as in Proposition 1.

\section{2-COCYCLE DEFORMED BICROSSED PRODUCTS}

Let $\mathbf{G}$ be a finite group and $\mathbf{H}, \mathbf{K}$ its subgroups such that $\mathbf{G}=\mathbf{K H}, \mathbf{K} \cap \mathbf{H}=\langle e\rangle$, that is, each element $g$ of $\mathbf{G}$ has a unique decomposition $g=t h$ for some $t \in \mathbf{K}, h \in \mathbf{H}$. $\mathbf{H}$ acts on $\mathbf{K}$ (as on a set) by $\{h \cdot t\}=\mathbf{K} \cap \mathbf{H} t h^{-1}$ and, likewise, $\mathbf{K}$ acts on $\mathbf{H}$ by $\{t \cdot h\}=$ $\mathbf{H} \cap \mathbf{K} h t^{-1}$. For any $h \in \mathbf{H}, t \in \mathbf{K}$ we have $(h \cdot t) h=(t \cdot h) t,(t \cdot h)^{-1}=(h \cdot t) \cdot h^{-1}$, $(h \cdot t)^{-1}=(t \cdot h) \cdot t^{-1}, h \cdot e=e, t \cdot e=e$, and $(t \cdot h) \cdot s t^{-1}=(h \cdot s)(h \cdot t)^{-1}$. From these identities it follows, in particular, that both $\mathbf{H}^{K}=\{h \in \mathbf{H} \mid t \cdot h=h \forall t \in \mathbf{K}\}$ and $\mathbf{K}^{H}=\{t \in \mathbf{K} \mid h \cdot t=t \forall h \in \mathbf{H}\}$ are subgroups of $\mathbf{H}$ and $\mathbf{K}$, respectively.

We now describe what is essentially a special case of the Kac double complex (see 
[5] or [8] for a more recent exposition). Let $k$ be a field such that both $|\mathbf{H}|$ and $|\mathbf{K}|$ are non-zero in $k$ and let $k^{*}$ be the multiplicative group of non-zero elements of $k$, viewed as a trivial $\mathbf{H}$ and $\mathbf{K}$ module. For $n, m=0,1, \ldots$ we consider $C^{n} C^{m}=$ $\left\{\phi:\left(\prod_{n} \mathbf{H}\right) \times\left(\prod_{m} \mathbf{K}\right) \rightarrow k^{*}\right\}$. Each $C^{n} C^{m}$ is an Abelian group with pointwise multiplication. Throughout this article all groups will be written multiplicatively, no matter if they are Abelian or not. We define coboundary maps $\partial_{H}^{n, m}: C^{n} C^{m} \rightarrow$ $C^{n+1} C^{m}$ and $\partial_{K}^{n, m}: C^{n} C^{m} \rightarrow C^{n} C^{m+1}$ by

$$
\begin{array}{r}
\partial_{H}^{n, m} \phi\left(h_{1}, \ldots, h_{n+1}, t_{1}, \ldots, t_{m}\right)=\phi\left(h_{2}, \ldots, h_{n+1}, h_{1}^{-1} \cdot\left(t_{1}, \ldots, t_{m}\right)\right) \\
\prod_{i=1}^{n} \phi\left(h_{1}, \ldots, h_{i-1}, h_{i} h_{i+1}, h_{i+2}, \ldots, h_{n+1}, t_{1}, \ldots, t_{m}\right)^{(-1)^{i}} \\
\phi\left(h_{1}, \ldots, h_{n}, t_{1}, \ldots, t_{m}\right)^{(-1)^{n+1}} \\
\partial_{K}^{n, m} \phi\left(h_{1}, \ldots, h_{n}, t_{1}, \ldots, t_{m+1}\right)=\phi\left(t_{1}^{-1} \cdot\left(h_{1}, \ldots, h_{n}\right), t_{2}, \ldots, t_{m+1}\right) \\
\prod_{i=1}^{m} \phi\left(h_{1}, \ldots, h_{n}, t_{1}, \ldots, t_{i-1}, t_{i} t_{i+1}, t_{i+2}, \ldots, t_{m+1}\right)^{(-1)^{i}} \\
\phi\left(h_{1}, \ldots, h_{n}, t_{1}, \ldots, t_{m}\right)^{(-1)^{m+1}},
\end{array}
$$

with the actions $\mathbf{H} \times \prod_{m} \mathbf{K} \rightarrow \prod_{m} \mathbf{K}$ and $\mathbf{K} \times \prod_{n} \mathbf{H} \rightarrow \prod_{n} \mathbf{H}$ defined as

$$
\begin{aligned}
& h \cdot\left(t_{1}, \ldots, t_{m}\right)=\left(\left(h \cdot t_{1}^{-1}\right)^{-1}, \ldots,\left(h \cdot\left(t_{1} \ldots t_{m-1}\right)^{-1}\right)\left(h \cdot\left(t_{1} \ldots t_{m}\right)^{-1}\right)^{-1}\right), \\
& t \cdot\left(h_{1}, \ldots, h_{n}\right)=\left(\left(t \cdot h_{1}^{-1}\right)^{-1}, \ldots,\left(t \cdot\left(h_{1} \ldots h_{n-1}\right)^{-1}\right)\left(t \cdot\left(h_{1} \ldots h_{n}\right)^{-1}\right)^{-1}\right) .
\end{aligned}
$$

In particular, the above action of $\mathbf{H}$ on $\mathbf{K}$ is induced from the action of $\mathbf{G}$ on its homogeneous space $\mathbf{G} / \mathbf{H} \cong \mathbf{K}$. Likewise for the action of $\mathbf{K}$ on $\mathbf{H}$. We denote orbits and stabilisers for these actions by $O_{H}(t), \mathbf{H}_{t}, O_{K}(h)$, and $\mathbf{K}_{h}$, respectively. We shall usually write $\partial_{H}^{n, m}=\partial_{H}$ and $\partial_{K}^{n, m}=\partial_{K}$. We have $\partial_{H} \partial_{H}=1, \partial_{K} \partial_{K}=1$, and $\partial_{H} \partial_{K}=\partial_{K} \partial_{H}$. The complex $\left\{C^{*} C^{m}, \partial_{H}\right\}$ is obviously isomorphic to the standard complex of inhomogeneous cochains of the group $\mathbf{H}$ with values in the $\mathbf{H}$-module $C^{0} C^{m}=\left\{\phi: \prod_{m} \mathbf{K} \rightarrow k^{*}\right\}$. We shall denote groups of coboundaries, cocycles, and cohomologies of this complex by $B^{*} C^{m}, Z^{*} C^{m}$, and $H^{*} C^{m}$, respectively. Likewise, the complex $\left\{C^{n} C^{*}, \partial_{K}\right\}$ is isomorphic to the standard complex of inhomogeneous cochains of the group $\mathbf{K}$ with values in the $\mathrm{K}$-module $C^{n} C^{0}=\left\{\phi: \prod_{n} \mathbf{H} \rightarrow k^{*}\right\}$. We denote groups of coboundaries, cocycles, and cohomologies of this complex by $C^{n} B^{*}, C^{n} Z^{*}$, and $C^{n} H^{*}$, respectively. We shall also use the notation $B^{n} Z^{m}=B^{n} C^{m} \cap C^{n} Z^{m}$, $Z^{n} Z^{m}=Z^{n} C^{m} \cap C^{n} Z^{m}$, and so on. 
Let $k(\mathbf{K})$ denote the algebra of $k$-valued functions on $\mathbf{K}$ with pointwise multiplication. Let $\gamma: \mathbf{H} \rightarrow \operatorname{Aut}(k(\mathbf{K}))$ be the action induced by the action of $\mathbf{H}$ on $\mathbf{K}$ and let $\mu \in C^{2} C^{1}$ be a normalised 2-cocycle for $\gamma$. That is, $\mu\left(h, f, g^{-1} \cdot t\right) \mu(g, h f, t)=$ $\mu(g, h, t) \mu(g h, f, t)$ and $\mu(g, h, t)=1$ whenever $g=e$ or $h=e$. We denote by $H_{\gamma}^{2}$ the subgroup of $C^{2} C^{1}$ consisting of all such functions. We consider the twisted crossed product algebra $k(\mathbf{K}) \rtimes_{\gamma, \mu} \mathbf{H}$. This algebra has a basis $\left\{p_{t} v_{h} \mid t \in \mathbf{K}, h \in \mathbf{H}\right\}$ such that

$$
p_{t} v_{h} p_{s} v_{g}=\delta_{t, h \cdot s} \mu(h, g, t) p_{t} v_{h g}
$$

with $\delta$ the Kronecker symbol. Similarly, let $\nu \in C^{1} C^{2}$ be a normalised 2-cocycle for the action $\sigma: \mathbf{K} \rightarrow \operatorname{Aut}(k(\mathbf{H}))$, induced by the action of $\mathbf{K}$ on $\mathbf{H}$. Again this means that $\nu\left(s^{-1} \cdot g, t, r\right) \nu(g, s, t r)=\nu(g, s, t) \nu(g, s t, r)$ and $\nu(h, s, t)=1$ whenever $s=e$ or $t=e$. We denote by $H_{\sigma}^{2}$ the subgroup of $C^{1} C^{2}$ consisting of all such functions. The twisted crossed product algebra $k(\mathbf{H}) \rtimes_{\sigma, \nu} \mathbf{K}$ has a basis $\left\{q_{h} z_{t} \mid h \in \mathbf{H}, t \in \mathbf{K}\right\}$ such that

$$
q_{g} z_{s} q_{h} z_{t}=\delta_{g, s \cdot h} \nu(g, s, t) q_{g} z_{s t} .
$$

Algebras $k(\mathbf{K}) \rtimes_{\gamma, \mu} \mathbf{H}$ and $k(\mathbf{H}) \rtimes_{\sigma, \nu} \mathbf{K}$ are dual to one another through the bilinear form

$$
\left\langle p_{t} v_{h}, q_{g} z_{s}\right\rangle=\delta_{t, h \cdot s} \delta_{g, s \cdot h}
$$

This duality determines a coassociative comultiplication

$$
\Delta: k(\mathbf{K}) \rtimes_{\gamma, \mu} \mathbf{H} \rightarrow\left(k(\mathbf{K}) \rtimes_{\gamma, \mu} \mathbf{H}\right) \otimes\left(k(\mathbf{K}) \rtimes_{\gamma, \mu} \mathbf{H}\right)
$$

such that

$$
\left\langle\Delta(a), b_{1} \otimes b_{2}\right\rangle=\left\langle a, b_{1} b_{2}\right\rangle
$$

for any $a \in k(\mathbf{K}) \rtimes_{\gamma, \mu} \mathbf{H}, b_{1}, b_{2} \in k(\mathbf{H}) \rtimes_{\sigma, \nu} \mathbf{K}$. On the basis this yields

$$
\Delta\left(p_{t} v_{h}\right)=\sum_{s \in K} \nu\left(\left(t \cdot h^{-1}\right)^{-1},\left(h^{-1} \cdot t\right)\left(h^{-1} \cdot s\right)^{-1}, h^{-1} \cdot s\right) p_{t s^{-1}} v_{\left(s \cdot h^{-1}\right)^{-1}} \otimes p_{s} v_{h} .
$$

$\Delta$ preserves the multiplication if and only if

$$
\begin{array}{r}
\frac{\nu\left(s \cdot h, s t^{-1}, t\right) \nu\left(\left(s \cdot g^{-1}\right)^{-1},\left(g^{-1} \cdot s\right)\left(g^{-1} \cdot t\right)^{-1}, g^{-1} \cdot t\right)}{\nu\left((s \cdot h)\left(s \cdot g^{-1}\right)^{-1},\left(g^{-1} \cdot s\right)\left(g^{-1} \cdot t\right)^{-1}, g^{-1} \cdot t\right)} \\
=\frac{\mu(h, g, h \cdot s)}{\mu\left(t \cdot h,\left(t \cdot g^{-1}\right)^{-1},(h \cdot s)(h \cdot t)^{-1}\right) \mu(h, g, h \cdot t)}
\end{array}
$$

for any $h, g \in \mathbf{H}, s, t \in \mathbf{K}$. If (1) holds then we say that the pair $(\mu, \nu)$ is matched. This implies that $\mu(h, g, t)=\nu(h, t, s)=1$ whenever $h=e, g=e, s=e$, or $t=e$. 
If $(\mu, \nu)$ are matched then we define a counit $\varepsilon: k(\mathbf{K}) \rtimes_{\gamma, \mu} \mathbf{H} \rightarrow k$ and an antipode $S: k(\mathbf{K}) \rtimes_{\gamma, \mu} \mathbf{H} \rightarrow k(\mathbf{K}) \rtimes_{\gamma, \mu} \mathbf{H}$ by

$$
\begin{aligned}
\varepsilon\left(p_{t} v_{h}\right) & =\delta_{t, e} \\
S\left(p_{t} v_{h}\right) & =\left[\mu\left(\left(t \cdot h^{-1}\right)^{-1}, t \cdot h^{-1}, t^{-1}\right) \nu\left(h,\left(h^{-1} \cdot t\right)^{-1}, h^{-1} \cdot t\right)\right]^{-1} p_{\left(h^{-1} \cdot t\right)^{-1} v_{t \cdot h}-1}
\end{aligned}
$$

to obtain a Hopf algebra structure on $k(\mathbf{K}) \rtimes_{\gamma, \mu} \mathbf{H}$. We shall denote this Hopf algebra by $\mathbf{K} \bowtie_{\mu, \nu} \mathbf{H}$. Clearly $\mathbf{H} \bowtie_{\nu, \mu} \mathbf{K}=\left(\mathbf{K} \bowtie_{\mu, \nu} \mathbf{H}\right)^{\circ}$, the dual Hopf algebra.

In order to describe some classes of solutions to (1) we now define two involutive maps: $C^{2} C^{1} \rightarrow C^{2} C^{1}, \phi \mapsto \widetilde{\phi}(h, g, t)=\phi\left(h, g, t^{-1}\right)^{-1}$, and $C^{1} C^{2} \rightarrow C^{1} C^{2}, \psi \mapsto$ $\widetilde{\psi}(h, t, s)=\psi\left(h^{-1}, t, s\right)$. Note that $\phi \in H_{\gamma}^{2}$ if and only if $\tilde{\phi}$ is a normalised element of $Z^{2} C^{1}$, and $\psi \in H_{\sigma}^{2}$ if and only if $\widetilde{\psi}$ is a normalised element of $C^{1} Z^{2}$. We define an automorphism $\Phi: C^{1} C^{2} \rightarrow C^{1} C^{2}$ by

$$
(\Phi \phi)(h, t, s)=\phi\left((t s)^{-1} \cdot h^{-1},\left(t^{-1} \cdot h^{-1}\right) \cdot s^{-1}, h^{-1} \cdot t^{-1}\right)
$$

It is easy to verify that $\Phi\left(C^{1} Z^{2}\right)=C^{1} Z^{2}, \Phi\left(C^{1} B^{2}\right)=C^{1} B^{2}, \Phi\left(Z^{1} C^{2}\right)=Z^{1} C^{2}$, and $\Phi\left(B^{1} C^{2}\right)=B^{1} C^{2}$. We can now rewrite identity (1) as

$$
\partial_{H} \Phi \widetilde{\nu}=\partial_{K} \widetilde{\mu}
$$

From (2) one immediately gets the following.

Proposition 1. If $\mathbf{G}=\mathbf{K H}$ is a matched pair of finite groups, $\mu \in H_{\gamma}^{2}$, $\nu \in H_{\sigma}^{2}, \widetilde{\mu} \in Z^{2} Z^{1}$, and $\widetilde{\nu} \in Z^{1} Z^{2}$, then the cocycles $(\mu, \nu)$ are matched.

In order to distinguish non-isomorphic Hopf algebras one may often calculate their intrinsic groups of group-like elements. For twisted bicrossed products this can be done with the help of the following Proposition 2. If $h \in \mathbf{H}$ and $t \cdot h=h$ for any $t \in \mathbf{K}$ then $h$ acts on $\mathbf{K}$ by automorphisms and $\nu(h, \cdot, \cdot)$ is an inhomogeneous 2-cocycle of $\mathbf{K}$ with values in the trivial $\mathrm{K}$-module $k^{*}$. We denote

$$
\begin{aligned}
& \mathbf{H}_{0}=\{h \in \mathbf{H} \mid t \cdot h=h, \forall t \in \mathbf{K} \text { and } \nu(h, \cdot, \cdot) \text { is a coboundry }\}, \\
& \mathbf{K}_{0}=\{t \in \mathbf{K} \mid h \cdot t=t, \forall h \in \mathbf{H} \text { and } \mu(\cdot, \cdot, t) \text { is a coboundry }\} .
\end{aligned}
$$

Both $\mathbf{H}_{0}$ and $\mathrm{K}_{0}$ are subgroups of $\mathrm{H}$ and $\mathrm{K}$, respectively. We denote $G\left(\mathrm{~K} \bowtie_{\mu, \nu} \mathbf{H}\right)=$ $\left\{a \in \mathbf{K} \bowtie_{\mu, \nu} \mathbf{H} \mid \Delta(a)=a \otimes a, a \neq 0\right\}$, the intrinsic group of group-like elements. The following proposition was originally proved in [3, Proposition 2.2]. The proof below is different and we believe much more transparent. It is also similar to the proof of Theorem 9 , below. 
PROPOSITION 2. There exist exact sequences

$$
\begin{aligned}
& \mathbf{1} \rightarrow \operatorname{Hom}\left(\mathbf{K}, k^{*}\right) \rightarrow G\left(\mathbf{K} \bowtie_{\mu, \nu} \mathbf{H}\right) \rightarrow \mathbf{H}_{0} \rightarrow \mathbf{1} \\
& \mathbf{1} \rightarrow \operatorname{Hom}\left(\mathbf{H}, k^{*}\right) \rightarrow G\left(\mathbf{H} \bowtie_{\nu, \mu} \mathbf{K}\right) \rightarrow \mathbf{K}_{0} \rightarrow \mathbf{1}
\end{aligned}
$$

Proof: Let $0 \neq a=\sum_{t, h} \lambda_{t, h} p_{t} v_{h} \in\left(\mathrm{K} \bowtie_{\mu, \nu} \mathbf{H}\right)$ for some $\lambda_{t, h} \in k$. Then $\Delta(a)=$ $a \otimes a$ if and only if

$$
\lambda_{s, h} \sum_{g \in H} \lambda_{t s^{-1}, g} v_{g}=\lambda_{t, h} \nu\left(\left(t \cdot h^{-1}\right)^{-1},\left(h^{-1} \cdot t\right)\left(h^{-1} \cdot s\right)^{-1}, h^{-1} \cdot s\right) v_{\left(s \cdot h^{-1}\right)^{-1}}
$$

for any $t, s \in \mathbf{K}, h \in \mathbf{H}$. Since $\left\{v_{g}\right\}$ are linearly independent this equality can only be satisfied if there exists exactly one $h \in \mathbf{H}$ such that $\lambda_{t, h} \neq 0$. For this $h$ we have $\lambda_{t} \stackrel{\text { def }}{=} \lambda_{t, h} \neq 0$ for any $t \in \mathbf{K}$. Hence, every element $a \in G\left(\mathbf{K} \bowtie_{\mu, \nu} \mathbf{H}\right)$ is of the form $a=\sum_{t} \lambda_{t} p_{t} v_{h}, h$ a fixed element of $\mathbf{H}, \lambda_{t} \neq 0$. We write $a=a_{\lambda, h}$. Now (3) yields

$$
\nu(h, x, y)=\lambda_{h \cdot x} \lambda_{h \cdot y} \lambda_{(h \cdot x)(h \cdot y)}^{-1}
$$

for any $x, y \in \mathbf{K}$, and $s \cdot h=h$ for any $s \in \mathbf{K}$. Thus $h \in \mathbf{H}_{0}$. Since $a_{\lambda, h} a_{\lambda^{\prime}, g}=$ $a_{\lambda^{\prime \prime}, h g}$ for any $h, g \in \mathbf{H}_{0}$ the map $a_{\lambda, h} \mapsto h$ gives a surjective homomorphism $\pi$ : $G\left(\mathbf{K} \bowtie_{\mu, \nu} \mathbf{H}\right) \rightarrow \mathbf{H}_{0}$. If $h=e$ then $\nu(h, x, y)=1$ by (1), and (4) implies that $\lambda \in \operatorname{Hom}\left(\mathbf{K}, k^{*}\right)$. Hence $\operatorname{ker}(\pi)=\operatorname{Hom}\left(\mathbf{K}, k^{*}\right)$, which gives the first exact sequence. The second one is constructed analogously.

We now describe two examples taking $k=\mathbb{C}$, the complex numbers. We denote by $\mathbf{S}_{n}, \mathbf{A}_{n}, \mathbf{Z}_{n}$, and $\mathbf{D}_{n}$ the symmetric, alternating, cyclic, and dihedral group, respectively.

EXAmple 3. There is a decomposition $\mathbf{S}_{4}=\mathbf{D}_{4} \mathbf{Z}_{3}, \mathbf{K}=\mathbf{D}_{4}=\left\langle\left(\begin{array}{llll}1 & 2 & 3 & 4\end{array}\right),\left(\begin{array}{ll}1 & 4\end{array}\right)\left(\begin{array}{ll}2 & 3\end{array}\right)\right\rangle$

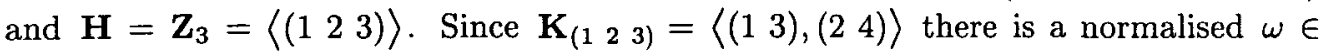

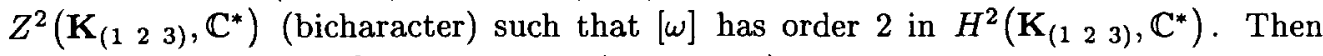

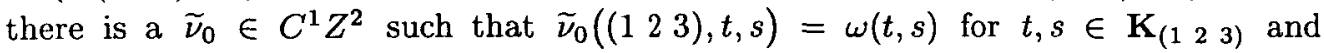
$\widetilde{\nu}_{0}(h, t, s)=1$ if $h=e, t=e$, or $s=e$. Since $\mathbf{K}^{H}=\langle(13)\rangle$ the restriction of $\widetilde{\nu}_{0}$ to $\mathbf{K}^{H}$ is a coboundary and there exists a $\phi \in C^{1} C^{1}$ such that $\phi(h, e)=\phi(e, t)=1$ and $\widetilde{\nu}=\widetilde{\nu}_{0}\left(\partial_{K} \phi\right)$ is 1 when restricted to $\mathbf{K}^{H}$. If $x \in \mathbf{K} \times \mathbf{K}$ and $\mathbf{K}_{x} \neq\langle e\rangle$ then $x \in \mathbf{K}^{H} \times \mathbf{K}^{H}$. Consequently, for any $x \in \mathbf{K} \times \mathbf{K}$ and $h \in \mathbf{H}$ our $\widetilde{\nu}$ is constant on $\left(\mathbf{H}_{x} h, x\right)$. This implies that $\widetilde{\nu} \in B^{1} C^{2}$ and, hence, $\widetilde{\nu}$ is in $Z^{1} Z^{2}$ and $[\widetilde{\nu}]$ is non-trivial in $C^{1} H^{2}$. Since $\mathbf{H}_{0}=1$ and $\mathbf{K}_{0}=\mathbf{K}^{H}$, by Proposition 2 we have $G\left(\mathbf{K} \bowtie_{\nu} \mathbf{H}\right) \cong \mathbf{Z}_{2} \times \mathbf{Z}_{2}$ and $G\left(\left(\mathbf{K} \bowtie_{\nu} \mathbf{H}\right)^{\circ}\right)$ is an extension of $\mathbf{Z}_{3}$ by $\mathbf{Z}_{2}$. Since $G\left(\left(\mathbf{K} \bowtie_{\nu} \mathbf{H}\right)^{\circ}\right)$ is generated

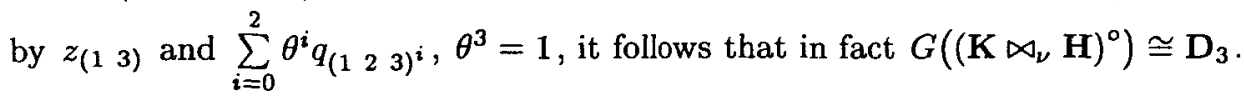


EXAMPLE 4. We consider PSL $\left(2, \mathbf{F}_{7}\right)=\mathbf{K H}$, where

$$
\begin{aligned}
& \mathbf{K}=\left\langle t=\left[\begin{array}{ll}
1 & 1 \\
0 & 1
\end{array}\right], s=\left[\begin{array}{ll}
2 & 0 \\
0 & 4
\end{array}\right]\right\rangle \cong \mathbf{Z}_{7} \rtimes \mathbf{Z}_{3}, \\
& \mathbf{H}=\left\langle g=\left[\begin{array}{ll}
2 & 1 \\
1 & 1
\end{array}\right], h=\left[\begin{array}{cc}
0 & 1 \\
-1 & 0
\end{array}\right]\right\rangle \cong \mathbf{D}_{4} .
\end{aligned}
$$

We are looking for a normalised $\widetilde{\mu} \in Z^{2} Z^{1}$. However, since the orders of $\mathbf{H}$ and $\mathbf{K}$ are relatively prime it sufficies to find $\tilde{\mu} \in Z^{2} B^{1}$. The action of $\mathbf{K}$ on $\mathbf{H} \backslash\{e\}$ is transitive and if $b, f$ are distinct elements of $\mathbf{H} \backslash\{e\}$ then $\mathbf{K}_{b} \cap \mathbf{K}_{f}=\langle e\rangle$. Since $\mathbf{K}_{(b, c)}=$ $\mathbf{K}_{b} \cap \mathbf{K}_{b c}, \mathbf{K}_{(b, c)} \neq\langle e\rangle$ implies $b=e, c=e$, or $c=b^{-1}$. Thus, if $\tilde{\mu}\left(b, b^{-1}, \mathbf{K}\right)=1$ then $\tilde{\mu} \in C^{2} B^{1}$. However, up to a factor in $B^{2} C^{1}$ any element of $Z^{2} C^{1}$ satisfies this requirement. Thus, any $\tilde{\mu} \in Z^{2} C^{1}$ such that $\tilde{\mu}(b, c, r)=1$ whenever $b=e, c=e$, $r=e$, or $c=b^{-1}$ yields a deformation of the bicrossed product in question. The action of $\mathbf{H}$ on $\mathbf{K} \backslash\{e\}$ has 5 orbits: $O_{H}(s), O_{H}(t), O_{H}\left(t^{2}\right), O_{H}\left(s t^{3}\right)$, and $O_{H}\left(s t^{5}\right)$, with point stabilisers $\mathbf{H}_{s}=\langle e\rangle, \mathbf{H}_{t}=\langle g h\rangle, \mathbf{H}_{t^{2}}=\left\langle g^{2} h\right\rangle, \mathbf{H}_{s t^{3}}=\left\langle g^{2}, h\right\rangle$, and $\mathbf{H}_{s t^{5}}=$ $\left\langle g^{2}, g h\right\rangle$. This implies that matched cocycles are generated by suitably normalised $\widetilde{\mu}_{1}$ and $\widetilde{\mu}_{2}$ such that the restriction of $\widetilde{\mu}_{1}$ to $\mathbf{H}_{s t^{3}}$ is non-trivial and its restriction to $\mathbf{H}_{s t^{5}}$ is trivial in $H^{2}\left(\mathbf{Z}_{2} \times \mathbf{Z}_{2}, \mathbb{C}^{*}\right)$, while the restrictions of $\tilde{\mu}_{2}$ to both $\mathbf{H}_{s t^{3}}$ and $\mathbf{H}_{s t^{5}}$ are nontrivial. One can verify that $\widetilde{\mu}_{1} \widetilde{\mu}_{2}$ yields a Hopf algebra isomorphic to the one resulting from $\tilde{\mu}_{1}$. Thus, in the present case there are two non-isomorphic deformations. Since $\mathbf{K}_{0}=\mathbf{H}_{0}=\mathbf{1}$, Proposition 2 gives $G\left(\mathbf{K} \bowtie_{\mu} \mathbf{H}\right) \cong \mathbf{Z}_{3}$ and $G\left(\left(\mathbf{K} \bowtie_{\mu} \mathbf{H}\right)^{\circ}\right) \cong \mathbf{Z}_{2} \times \mathbf{Z}_{2}$ for any $\mu$.

\section{PSEUDOCOCYCLE DEFORMED BICROSSED PRODUCTS}

The comultiplication in $\mathbf{K} \bowtie \mathbf{H}$ can be also twisted with the help of a pseudococycle. The following Definition 5 and Proposition 6 are borrowed from [1]. For convenience we denote $\mathbf{A}=\mathbf{K} \bowtie \mathbf{H}$.

Definition 5: An invertible element $\Omega \in \mathbf{A} \otimes \mathbf{A}$ is a pseudococycle if the following conditions hold:

1. $(i d \otimes \Delta)\left(\Omega^{-1}\right)\left(I \otimes \Omega^{-1}\right)(\Omega \otimes I)(\Delta \otimes i d)(\Omega)$ commutes with $(\Delta \otimes i d) \Delta(\mathbf{A})$,

2. $(\varepsilon \otimes i d)(\Omega),(i d \otimes \varepsilon)(\Omega), m(S \otimes i d)(\Omega)$, and $m(i d \otimes S)(\Omega)$ are in the centre of $\mathbf{A}$.

Proposition 6. If $\Omega$ is a pseudococycle then $\mathbf{A}$ with a new comultiplication

$$
\Delta_{\Omega}(x)=\Omega \Delta(x) \Omega^{-1}
$$


(and old multiplication, counit, and antipode) is a Hopf algebra.

The Hopf algebra $\mathbf{A}$ with the new comultiplication as in Proposition 6 is denoted by $\mathbf{A}_{\Omega}$. In general finding all possible pseudococycles is an exceedingly difficult problem. In order to be able to construct concrete new examples of deformed bicrossed products we consider below a special class of pseudococycles of the form

$$
\Omega=\sum_{t, s \in K} \omega(t, s) p_{t} v_{\alpha(s)} \otimes p_{s} v_{\beta(t)}
$$

with $\alpha, \beta: \mathbf{K} \rightarrow \mathbf{H}, \omega: \mathbf{K} \times \mathbf{K} \rightarrow k^{*}$. If $\alpha(t)=\beta(t)=e$ for any $t \in \mathbf{K}$ then the resulting Hopf algebra $\mathbf{A}_{\Omega}$ is isomorphic to $\mathbf{K} \bowtie_{\nu} \mathbf{H}$ for a suitable cocycle $\nu$. We restrict our considerations to the case when both $\alpha$ and $\beta$ are $\mathbf{H}$-invariant, that is,

$$
\alpha(h \cdot t)=\alpha(t) \text { and } \beta(h \cdot t)=\beta(t)
$$

for any $h \in \mathbf{H}$ and $t \in \mathbf{K}$. Condition (6) implies that an $\Omega$ as in (5) is invertible with

$$
\Omega^{-1}=\sum_{t, s \in K} \omega^{-1}(\alpha(s) \cdot t, \beta(t) \cdot s) p_{t} v_{\alpha^{-1}(s)} \otimes p_{s} v_{\beta^{-1}(t)}
$$

We only deal with a more managable case when the group $\mathbf{G}$ is a semi-direct product $\mathbf{K} \rtimes \mathbf{H}$ and $\mathbf{H}$ is Abelian. We further require that $\mathbf{K}$ acts on itself through $(t, s) \mapsto \alpha(t) \cdot s$ and $(t, s) \mapsto \beta(t) \cdot s$. For these two maps to be true actions we must have

$$
\begin{gathered}
\alpha(t) \alpha(s) \alpha^{-1}(t s) \cdot r=r \\
\beta(t) \beta(s) \beta^{-1}(t s) \cdot r=r
\end{gathered}
$$

for any $r, s, t \in \mathbf{K}$. If $\alpha$ and $\beta$ are as above then we say that they are matched if

$$
\alpha(t) \beta(t) \cdot t=t
$$

for any $t \in \mathbf{K}$.

Assuming that $\alpha$ and $\beta$ satisfy (6) and (7) we now define a cochain complex $\left\{F^{*}, \partial\right\}$, with $\partial=\partial_{\alpha, \beta}^{*}$, as follows. We set $F^{n}=\left\{\omega: \prod_{n} \mathbf{K} \rightarrow k^{*}\right\}$ and consider each $F^{n}$ a left $\mathbf{H}$-module with the action $h \cdot \omega\left(t_{1}, \ldots, t_{n}\right)=\omega\left(h \cdot t_{1}, \ldots, h \cdot t_{n}\right)$. We define the coboundary maps by

$$
\begin{aligned}
\partial^{n} \omega\left(t_{1}, \ldots, t_{n+1}\right)=\left(\alpha\left(t_{n+1}\right) \cdot \omega\right)\left(t_{1}, \ldots, t_{n}\right)^{(-1)^{n+1}}\left(\beta\left(t_{1}\right) \cdot \omega\right)\left(t_{2}, \ldots, t_{n+1}\right) \\
\prod_{i=1}^{n} \omega\left(t_{1}, \ldots, t_{i-1}, t_{i} t_{i+1}, t_{i+2}, \ldots, t_{n+1}\right)^{(-1)^{i}} .
\end{aligned}
$$


We denote the corresponding groups of cocycles, coboundaries, and cohomologies by $Z^{*}, B^{*}$, and $H^{*}$, respectively. We also define a bijection $F^{2} \rightarrow F^{2}, \omega \mapsto \widetilde{\omega}$, by

$$
\widetilde{\omega}(t, s)=\omega(\alpha(s) \cdot t, \beta(t) \cdot s) .
$$

It turns out (see Theorem 7 below) that for $\Omega$ as in (5) to be a pseudococycle it is necessary that $\widetilde{\omega}$ is a 2-cocycle, that is, $\widetilde{\omega} \in Z^{2}$. For $\omega \in F^{2}$ we say that $\omega$ is $\mathbf{H}$ normalised if the following three functions (of $t \in \mathbf{K}$ ): $\omega(e, t), \omega(t, e)$, and $\omega\left(t, t^{-1}\right)$, are $\mathbf{H}$-invariant.

Theorem 7. Let $\mathbf{G}=\mathbf{K} \rtimes \mathbf{H}$ be a finite group with $\mathbf{H}$ Abelian. Let $\alpha, \beta$ : $\mathbf{K} \rightarrow \mathbf{H}$ be such that (6), (7), and (8) hold, and let $\omega \in F^{2}$ be $\mathbf{H}$-normalised and such that $\widetilde{\omega} \in Z^{2}$. Then the corresponding $\Omega$ as in (5) is a pseudococycle in the sense of Definition 5.

Proof: Suppose that $\alpha$ and $\beta$ satisfy (6) and the corresponding $\Omega$ is as in (5). Let $U=(i d \otimes \Delta)\left(\Omega^{-1}\right)\left(I \otimes \Omega^{-1}\right)(\Omega \otimes I)(\Delta \otimes i d)(\Omega)$. To simplify the notation for any $x, y, z \in \mathbf{K}$ we denote $D=D(x, y, z)=\left(\alpha^{-1}\left(y z^{-1}\right) \alpha(y) \cdot x\right)\left(\alpha(z) \cdot y z^{-1}\right)$. We have

$$
\begin{aligned}
U=\sum_{x, y, z \in K} \eta(x, y, z) p_{x} v_{\alpha\left(y z^{-1}\right) \alpha^{-1}(y) \alpha(D, z)} & \\
& \otimes p_{y z^{-1}} v_{\alpha^{-1}(z) \alpha(D, z)} \otimes p_{z} v_{\beta^{-1}\left(y z^{-1}\right) \beta^{-1}(x) \beta(D, z)}
\end{aligned}
$$

where

$$
\begin{aligned}
& \eta(x, y, z)=\omega\left(D, \beta\left(y z^{-1}\right) \beta(x) \cdot z\right) \omega\left(\alpha(y) \cdot x, \alpha(z) \beta(x) \cdot y z^{-1}\right) \\
& \omega^{-1}\left(\alpha(z) \beta(x) \cdot y z^{-1}, \beta\left(y z^{-1}\right) \beta(x) \cdot z\right) \omega^{-1}(\alpha(y) \cdot x, \beta(x) \cdot y) .
\end{aligned}
$$

If $t \in \mathbf{K}$ then $U$ commutes with $(\Delta \otimes i d) \Delta\left(p_{t}\right)=\sum_{r, s \in K} p_{t s^{-1}} \otimes p_{s r-1} \otimes p_{r}$ if and only if

$$
\begin{aligned}
& z y^{-1}\left(\alpha^{-1}(z) \alpha^{-1}\left(y z^{-1}\right) \alpha(y) \cdot x^{-1}\right) x y \\
& \quad=\beta^{-1}\left(\left(\alpha^{-1}(z) \alpha^{-1}\left(y z^{-1}\right) \alpha(y) \cdot x\right) y z^{-1}\right) \beta\left(y z^{-1}\right) \beta(x) \cdot z
\end{aligned}
$$

for any $x, y, z \in \mathbf{K}$. Condition (7) implies that the above identity holds.

If $h \in \mathbf{H}$ then $U$ commutes with $(\Delta \otimes i d) \Delta\left(v_{h}\right)=v_{h} \otimes v_{h} \otimes v_{h}$ if and only if the function $\eta$ is $h$-invariant, that is, $h \cdot \eta=\eta$. This is the case if $\eta(x, y, z)=1$ for any $x, y, z \in \mathbf{K}$. If $\alpha$ and $\beta$ satisfy (7) then $\eta \equiv 1$ if and only if $\partial \widetilde{\omega}=1$. Thus, we have shown that the $\Omega$ satisfies condition 1 of Definition 5. On the other hand if $\alpha$ and $\beta$ are matched and $\omega$ is $\mathbf{H}$-normalised then it easily follows that condition 2 of Definition 5 holds true as well. 
Under the hypothesis of Theorem 7 we have

$$
\Delta_{\Omega}\left(p_{t} v_{h}\right)=\sum_{s \in K} \omega(W, s) \omega^{-1}\left(h^{-1} \cdot W, h^{-1} \cdot s\right) p_{W} v_{h} \otimes p_{s} v_{h}
$$

with $W=(\alpha(s) \cdot t)\left(\beta^{-1}(t) \cdot s^{-1}\right)$, for any $t \in \mathbf{K}, h \in \mathbf{H}$.

Proposition 8. Let $\mathbf{G}=\mathbf{K} \rtimes \mathbf{H}$ be a finite group with $\mathbf{H}$ Abelian. Let $\alpha, \beta$ : $\mathbf{K} \rightarrow \mathbf{H}$ be such that (6), (7), and (8) hold, and let $\omega_{1}, \omega_{2} \in F^{2}$ be $\mathbf{H}$-normalised and such that $\widetilde{\omega_{1}}, \widetilde{\omega_{2}} \in Z^{2}$. Let $\Omega_{i}, i=1,2$, be the pseudococycles as in (5) corresponding to $\omega_{1}$ and $\omega_{2}$, respectively. If $\left[\widetilde{\omega_{1}}\right]=\left[\widetilde{\omega_{2}}\right]$ in $H^{2}$ then the Hopf algebras $\mathbf{A}_{\Omega_{1}}$ and $\mathbf{A}_{\Omega_{2}}$ are isomorphic.

Proof: Let $\mathbf{A}_{\Omega_{1}}=\operatorname{span}\left\{p_{t} v_{h} \mid h \in \mathbf{H}, t \in \mathbf{K}\right\}$ and $\mathbf{A}_{\Omega_{2}}=\operatorname{span}\left\{P_{t} V_{h} \mid h \in\right.$ $\mathbf{H}, t \in \mathbf{K}\}$. Let $\phi: \mathbf{A}_{\Omega_{1}} \rightarrow \mathbf{A}_{\Omega_{2}}$ be the map defined by $\phi\left(p_{t} v_{h}\right)=\lambda(t) \lambda^{-1}\left(h^{-1} \cdot t\right) P_{t} V_{h}$. Then $\phi$ is an algebra isomorphism such that $(\phi \otimes \phi) \Delta_{\Omega_{1}}=\Delta_{\Omega_{2}} \phi$ and, thus, $\phi$ is a Hopf algebra isomorphism.

As before in order to distinguish non-isomorphic Hopf algebras $\mathbf{A}_{\Omega}$ arising from the construction in Theorem 7 we determine below the intrinsic group of group-like elements $G\left(\mathbf{A}_{\Omega}\right)=\left\{x \in \mathbf{A}_{\Omega} \backslash\{0\} \mid \Delta_{\Omega}(x)=x \otimes x\right\}$. To this end with the hypothesis of Theorem 7 in place we define

$$
\mathbf{H}_{0}=\left\{h \in \mathbf{H} \mid[h \cdot \widetilde{\omega}]=[\widetilde{\omega}] \text { in } H^{2}\right\}
$$

a subgroup of $\mathbf{H}$. Somewhat analogously to Proposition 2 we have the following.

THEOREM 9. Under the hypothesis of Theorem 7 there exists a short exact sequence

$$
\mathbf{1} \rightarrow Z^{1} \rightarrow G\left(\mathrm{~A}_{\Omega}\right) \rightarrow \mathbf{H}_{0} \rightarrow \mathbf{1}
$$

Proof: Let $\lambda(t, f) \in k, t \in \mathbf{K}, f \in \mathbf{H}$, and $0 \neq x=\sum_{t, f} \lambda(t, f) p_{t} v_{f}$ be such that $\Omega \Delta(x)=(x \otimes x) \Omega$. By expanding $\Omega$ and $x$ and simplifying we see that this equality holds if and only if

$$
\lambda(s, f) \sum_{g \in H} \omega\left(g^{-1} \cdot t, g^{-1} \cdot s\right) \lambda(t, g) v_{g}=\lambda\left(\left(\alpha^{-1}(s) \cdot t\right)\left(\beta^{-1}(t) \cdot s\right), f\right) \omega(t, s) v_{f}
$$

for any $t, s \in \mathbf{K}, f \in \mathbf{H}$. For (9) to be satisfied it is necessary that there is a unique $h \in \mathbf{H}$ such that $\lambda(t, g)=0$ if $g \neq h$. Thus, writing $\lambda(t)=\lambda(t, h)$, we have $x=$ $\sum_{t \in K} \lambda(t) p_{t} v_{h}$ and $(9)$ is equivalent to

$$
\frac{\lambda\left(\left(\alpha^{-1}(s) \cdot t\right)\left(\beta^{-1}(t) \cdot s\right)\right)}{\lambda(s) \lambda(t)}=\frac{\omega\left(h^{-1} \cdot t, h^{-1} \cdot s\right)}{\omega(t, s)}
$$


for any $t, s \in \mathbf{K}$. Substituting in (10) $x$ for $\alpha^{-1}(s) \cdot t, y$ for $\beta^{-1}(t) \cdot s$, and using (6) we see that $(10)$ is equivalent to

$$
h \cdot \widetilde{\omega}=(\partial \lambda) \tilde{\omega} .
$$

Thus $h \in \mathbf{H}_{0}$ and $\pi: x \mapsto h$ gives a surjective homomorphism $\pi: G\left(\mathbf{A}_{\Omega}\right) \rightarrow \mathbf{H}_{\mathbf{0}}$. If $\pi(x)=e$ then $\partial \lambda \equiv 1$ and hence $\operatorname{ker} \pi \cong Z^{1}$.

\section{REFERENCES}

[1] M. Enock and L. Vainerman, 'Deformation of the Kac algebra by an Abelian subgroup', Comm. Math. Phys. 178 (1996), 571-596.

[2] J.H. Hong and H. Kosaki, 'The group of one dimensional bimodules arising from composition of subfactors', J. Math. Soc. Japan (to appear).

[3] J.H. Hong and W. Szymański, 'Composition of subfactors and twisted bicrossed products', J. Operator Theory 37 (1997), 281-302.

[4] M. Izumi and H. Kosaki, 'Finite-dimensional Kac algebras arising from certain group actions on a factor', Internat. Math. Res. Notices 8 (1996), 357-370.

[5] G.I. Kac, 'Extensions of groups to ring groups', Math. USSR-Sb. 5 (1968), 451-474.

[6] S. Majid, Foundations of quantum group theory (Cambridge University Press, Cambridge, 1995).

[7] A. Masuoka, 'Calculations of some groups of Hopf algebra extensions', J. Algebra 191 (1997), 568-588.

[8] A. Masuoka, 'Twisted forms and cohomology of Hopf algebra extensions', (preprint).

[9] D. Nikshych, ' $K_{0}$-rings and twisting of finite dimensional semisimple Hopf algebras', (preprint).

[10] L. Vainerman, '2-cocycles and twisting of Kac algebras', Comm. Math. Phys. 191 (1998), 397-721.

[11] S. Wang, 'Rieffel type discrete deformation of finite quantum groups', (preprint).

Department of Applied Mathematics

Korea Maritime University

Pusan 606-791

Korea

e-mail: hongjh@hanara.kmaritime.ac.kr
Department of Mathematics

The University of Newcaslte

Newcastle NSW 2308

Australia

e-mail: wojciech@frey.newcastle.edu.au 